\title{
Operadores logísticos
}

\author{
Logistics Operators
}

\section{Rafael Granillo-Macías ${ }^{a}$, Isidro J. González-Hernández ${ }^{b}$, Francisca Santana-Robles ${ }^{c}$}

\begin{abstract}
:
This paper exposes the relevance and characteristics of logistics operators within a supply chain. A logistics operator is a provider that offers services mainly in storage and distribution operations representing a strategic advantage for the company that hires it, facilitating delivery to the customer from the point of departure to the point of delivery with security, flexibility, collaboration And control. Currently the sector of logistics providers is in full growth as shown by the progress made by Mexico in the creation of organizations that group the different logistics operators with the purpose of establishing quality standards.
\end{abstract}

Keywords:

Logistic, logistics operators, supply chain, outsourcing

\section{Resumen:}

En este trabajo se expone la relevancia y características de los operadores logísticos dentro de una cadena de suministro. Un operador logístico es un proveedor que ofrece servicios principalmente en las operaciones de almacenamiento y distribución representando una ventaja estratégica para la empresa que lo contrata, facilitando la entrega hacia el cliente desde el punto de salida hasta el punto de entrega con seguridad, flexibilidad, colaboración y control. Actualmente el sector de los proveedores logísticos se encuentra en pleno crecimiento como lo muestra los avances que México ha realizado para la creación de organismos que agrupen a los diferentes operadores logísticos con el propósito de establecer estándares de calidad.

\section{Palabras Clave:}

Logística, operador logístico, cadena de suministro, subcontratación

\section{Introducción}

La creciente competitividad en el mundo a partir de los años setentas motivo a muchas empresas a modificar sus modelos de negocio, emigrando a la tendencia de concentrarse en su "competencia central", donde muchas de estas llegaron a la conclusión que tanto las actividades de transporte, almacenamiento y manejo de materiales no eran parte de sus funciones estratégica, por los que podían ser delegadas a un tercero.
Como consecuencia de que prácticamente no existían empresas con servicios integrados de almacenamiento y transporte en el mercado para hacer outsourcing, el ruido social de despedir a muchos empleados que se desempeñaban en estas áreas, y el miedo a pasar a un tercero actividades particulares y sensibles de la empresa, llevaron a muchas empresas a constituir estas actividades como una empresa proveedora de servicios y posteriormente traspasar su propiedad a sus propios empleados, o poner a competir dicha nueva empresa con el mercado, dando origen al concepto masivo de "operador logístico".

\footnotetext{
a Autor de Correspondencia, Universidad Autónoma del Estado de Hidalgo, Escuela Superior de Ciudad Sahagún, ORCID: 0000-0002-1015667X, Email: rafaelgm@uaeh.edu.mx

b Universidad Autónoma del Estado de Hidalgo, Escuela Superior de Ciudad Sahagún, ORCID: 0000-0002-3301-9790, Email: profe_7739@uaeh.edu.mx 
Un operador logístico surgió como una empresa que ofrece básicamente dos servicios principales: el almacenamiento y transporte de productos, y que con el tiempo han agregado una serie de servicios complementarios o inmediatos que por encargo de un cliente son diseñados dentro de la cadena de suministro, como por ejemplo: servicios aduanero, re embalaje, etiquetado, envasado entre otros. Estos servicios pueden ser ofrecidos ya sea a través de activos propios en camiones y bodegas, o a través de la integración de activos en outsourcing. 1, 2

Actualmente la integración de estos servicios se efectúa a través de la adecuación y especialización de sistemas de información que se acoplan a los clientes, que permiten sentir al operador logístico como un área más del mismo cliente.

\section{Operadores logísticos}

A partir del enfoque en el que la especialización resultaba una alternativa para potenciar la entrega de un producto o servicio de calidad y además concedía una ventaja competitiva ante la competencia, surge el concepto de la subcontratación como antecedente a los operadores logísticos. La subcontratación se define como la contratación que una empresa hace de otra empresa, para que esta última realice parte de los servicios por los que la primera ha sido contratada directamente. 2

Un operador logístico se define entonces como la empresa que soportan el transporte y manejo de materiales desde el punto de salida en un origen hasta el punto de llegada en un destino. 1

La Asociación Española de Empresas de Almacenaje y Distribución Física (ANADIF) precisa que un operador logístico es aquella empresa que por encargo de su cliente diseña los procesos de una o varias fases de su cadena de suministro, organiza, gestiona y controla dichas operaciones utilizando para ello infraestructuras físicas, tecnología y sistemas de información, propios o ajenos, independientemente de que preste o no los servicios con medios propios o subcontratados. En este sentido, el operador logístico responde directamente ante su cliente de los bienes y de los servicios adicionales acordados en relación con éstos y es su interlocutor directo. 3,4

En cuanto a las funciones que realiza un operador logístico dentro de la cadena de suministro se encuentran:
- Procesamiento de pedidos: que son actividades relativas a la recolección, comprobación y envió de órdenes de compra.

- Manejo de materiales: determinan que medios materiales y procedimientos se han de utilizar para mover los productos dentro de almacenes y entre estos y los puntos de venta.

- Servicios de almacenamiento: encargado de seleccionar el emplazamiento, la dimensión y las características de los almacenes.

- Servicios de transporte de los productos: incluyen los servicios con mejores plazos de entrega y más rápido y fácil acceso a la información, decidir qué medios de transporte a utilizar y la elaboración de la ruta.

- Servicios complementarios: etiquetaje, embalaje, facturación, cobros, montaje.

- Actividades de gestión: organización de rutas, gestiones aduaneras, controles de cantidad y calidad de productos, gestión de stocks, gestión de caducidades de productos.

- Control de inventarios: determinación de la cantidad de productos que se deben tener disponibles para entregar a un posible comprador, estableciendo también la periodicidad de los pedidos.

\subsection{Clasificación de los operadores logísticos}

De manera general se distinguen cuatro tipos de operadores logísticos (tabla 1): 1PL, 2PL, 3PL y 4PL donde PL hace referencia a "party logistics" es decir, "proveedor de logística".

- 1PL (First Party Logistics), son operadores caracterizados por ofrecer servicios únicamente de transporte de mercancías a las empresas. Este tipo de operadores son el primer paso para la subcontratación de actividades que no forman parte de la competencia esencial del negocio.

- 2PL (Second Party Logistics), en este nivel se encuentra los operadores que ofrecen en conjunto el transporte y almacenaje de mercancía, siendo un servicio añadido que repercute positivamente en la administración del flujo de materiales.

- 3PL (Third Party Logistics), la característica de este operador es que se encarga de la mayoría de las operaciones logísticas en la empresa. Generalmente están especializados en la integración y personalización de los servicios relacionados con el almacenaje, la administración de inventarios, la preparación de pedidos y el transporte.

- 4PL (Fourth Party Logistics), la diferencia con un 3PL es que este tipo de operador crea una unión en la empresa y los diferentes proveedores de servicios logísticos, resultando en la administración de todas 
las áreas de la cadena de suministros incluye a los clientes y proveedores.

\begin{tabular}{|l|l|l|l|l|}
\cline { 2 - 5 } \multicolumn{1}{c|}{} & Transporte & Almacenaje & $\begin{array}{l}\text { Administración } \\
\text { de inventarios }\end{array}$ & $\begin{array}{l}\text { Preparación } \\
\text { de pedidos }\end{array}$ \\
\hline 1PL & $\mathrm{X}$ & & & \\
\hline 2PL & $\mathrm{X}$ & $\mathrm{X}$ & & $\mathrm{X}$ \\
\hline 3PL & $\mathrm{X}$ & $\mathrm{X}$ & $\mathrm{X}$ & $\mathrm{X}$ \\
\hline 4PL & Administración total de la cadena de suministro \\
\hline
\end{tabular}

Tabla 1. Clasificación de operadores logísticos

\subsection{Ventajas y desventajas de la contratación de operadores logísticos}

Entre las ventajas de contratar operadores logísticos están:

- Enfoque estratégico más claro dentro de la empresa.

- Se mejora la asignación de recursos.

- Disponibilidad de los conocimientos de los expertos en aspectos de logística.

- Control de costos a largo plazo.

- Mejorar la orientación cliente/proveedor.

- El acceso a nuevos mercados.

- Aplicación de talento y de recursos de la empresa en áreas clave de la organización.

Entre las principales desventajas de la contratación de operadores logísticos están:

- Vulnerabilidad en cuanto a la confidencialidad de los datos de la empresa.

- $\quad$ Se puede crear redundancias en los procesos de negocio.

- El operador logístico se puede declarar en quiebra o pasar por dificultades importantes.

- El cliente puede no ser atendido con la dedicación necesaria, debido a que el operador cuente con más clientes que atender.

\section{Aspectos a considerar para la contratación}

Debido a que la competencia obliga a las empresas a poner especial atención no únicamente en sus actividades de negocio, sino también en sus proveedores clave, ya que la calidad y precio del producto final depende de la interacción de los suministros y servicios que ofrecen múltiples proveedores, es necesario elegir proveedores confiables, estratégicos y con enfoques de asociación a largo plazo. 5
DHL empresa especialista en logística a nivel mundial elaboro una guía para ayudar a empresas de bienes y productos de consumo a tener un proceso más riguroso para la elección de un proveedor de soluciones logísticas, con la finalidad de agregar valor real al negocio más allá de limitarse a ser una fuente de mano de obra o transporte. 4

El primer elemento determinante a considerar es la infraestructura con la que cuenta el operador logístico, ya que a través de esta se pueden lograr economías de escala al compartir recursos con otros productos que se muevan en el mismo canal. Un segundo aspecto es la relevancia que tiene la experiencia del proveedor para la empresa que lo desea contratar, la experiencia se considera importante de acuerdo a la dinámica de los bienes y servicios que se mueve a lo largo de la cadena de suministro.

Con el propósito de asegurar que el proveedor logístico opere con gran eficiencia y fiabilidad manteniendo la capacidad de reaccionar a los cambios en la demanda o a las oportunidades que presenten las diferentes temporadas se sugiere como tercer elemento el evaluar si el proveedor desarrolla los procesos necesarios para garantizar la fiabilidad y calidad de los servicios que ofrece.

Otro aspecto es la mano de obra altamente calificada, ya que la cadena de suministro se beneficiara de este aspecto. Después, se debe considerar la amplitud en el portafolio de soluciones y estabilidad operativa con la que pueda llegar a contar el proveedor logístico. Finalmente la "personalización" en las soluciones que se puedan crear para la empresa, el proveedor debe ser capaz de adaptar las soluciones que ofrece a las necesidades de cada cliente, canal y país.

\section{Operadores logísticos en México}

En México concurren operadores logísticos con diferentes niveles de desarrollo, destacando que es muy restringida la producción de servicios de valor agregado debido principalmente a la resistencia a utilizar terceros en algunas operaciones, en particular en almacenes de producto terminado. 5

En México existe un directorio de operadores logísticos (figura 1) el cual fue elaborado con el apoyo de las delegaciones federales de la secretaría de economía. Este directorio contiene información básica de los diferentes prestadores de servicios establecidos en las diferentes entidades federativas del país, mismo que se actualiza de forma trimestral y se encuentra disponible 
para su consulta en la página del Sistema Nacional de Información e Integración de Mercados, a la fecha existen registradas en este directorio 415 empresas que brindan sus servicios como operadores logísticos en la República Mexicana. 6

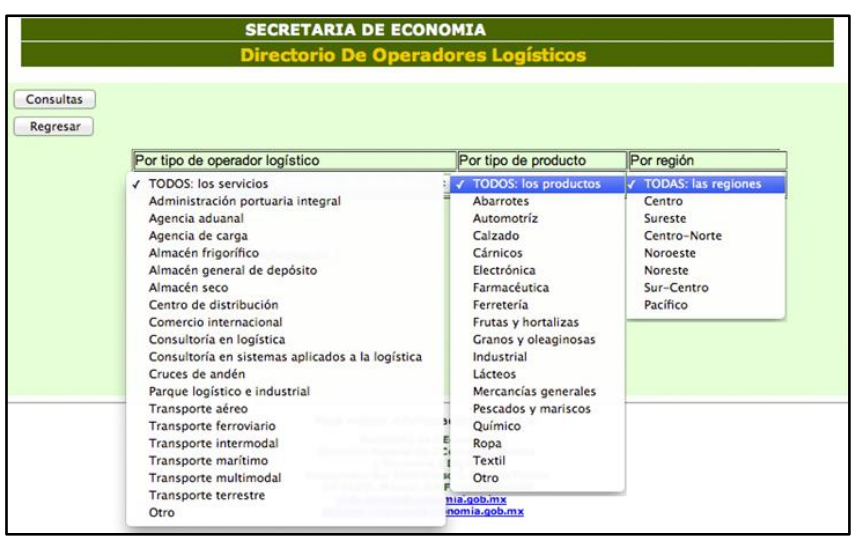

Figura 1: Directorio de operadores logísticos

Este directorio provee también información sobre los tipos de operadores logísticos, el tipo de producto y su ubicación geográfica dentro del país.

\subsection{Asociación de operadores logísticos de México (AOLM)}

En el 2013, un grupo de empresas especializadas en operaciones logísticas formado por DHL Supply Chain, FedEx, Accel, Grupo Eduardo Diaz, Grupo Porteo, Grupo SID, Onest Logistics, Rosch Transporte y Ryder, constituyeron la Asociación de Operadores Logísticos de México (AOLM) con el objetivo de intermediar con la autoridad en la toma de decisiones en lo relativo al manejo de mercancías.

El reto principal bajo el cual surgió esta asociación fue el de promover el acercamiento con las autoridades para convertirse en el representante del sector integrando a diferentes operadores logísticos a lo largo del país. 7

Esta asociación se formó también con el propósito de establecer requisitos mínimos de calidad en el servicio para generar confianza en el usuario, y que este sea capaz de distinguir entre un operador logístico y un proveedor de un servicio de la cadena. La misión de esta asociación es ser un organismo que represente los intereses legítimos de sus asociados, orientada a impulsar el desarrollo y la competitividad de la actividad logística en México, aportando conocimiento y buenas prácticas de operación para satisfacer plenamente las necesidades de sus clientes, generando valor a sus representados y al país. 7

Las autoridades involucradas en la AOLM son la Secretaria de Comunicaciones y Transporte (SCT), Secretaría de Hacienda y Crédito Público (SHCP) y Secretaría de Seguridad Pública (SSP).

\section{Conclusiones}

Sin duda el cliente a cada momento se vuelve más exigente en cuanto a sus requerimientos en la calidad, el tiempo de entrega y el precio de los productos y servicios que demanda, es por ello que las empresas envueltas en un entorno de alta competitividad buscan continuamente oportunidades para la mejora en sus procesos y en la respuesta a sus clientes.

El desarrollo de las funciones logísticas a lo largo de la cadena de suministro representa una fuente de valor para los clientes gozando como principales efectos la reducción de costos y tiempos de entrega.

Una ventaja competitiva en el mercado es el tener un amplio conocimiento sobre el canal donde viajamos como empresa a pesar de esto actualmente existen muchas empresas que no tienen conocimientos precisos sobre el canal de distribución, esto les cuesta mucho dinero, tiempo y esfuerzo, en cambio los operadores logísticos son actores sumamente importantes en la industria logística, son empresas que facilitan el transporte y manejo de la carga desde el punto de salida hasta el punto de entrega, manejándolo con seguridad, flexibilidad, colaboración y control, entre otros aspectos benéficos.

Considerando estas exigencias de los clientes, las empresas optan por contratar total o parcialmente sus servicios, con el fin de aprovechar de la infraestructura y economía de escala que maneja el operador logístico, y conseguir mejor rentabilidad.

México es un país de muchas oportunidades para este sector que se encuentra en pleno crecimiento, y ya ha dado los primeros pasos con una organización que agrupa a los principales operadores logísticos. Aunque muchas empresas están aún renuentes sobre los beneficios de los proveedores de logística, el mismo mercado está impulsando a dar el siguiente paso para visualizar nuevos portafolios de soluciones que ofrecen los especialistas de la logística con el propósito de contribuir en mayor escala al desempeño de la cadena de suministro como un todo. 


\section{Referencias}

[1] Marasco, Alessandra. Third-party logistics: A literature review. International Journal of Production Economics. 2008; 113(1): 127-147.

[2] Solís A. ¿Qué es un operador logístico? The passenger transporte y logística. Extraido del sitio web icexpasseger.blogspot.bg/2013/03/quees-un-operador-logistico.html el 03/07/2013; 2013.

[3] Simchi-Levi, D., Kaminsky, Ph., Simchi-Levi, E. Managing the Supply Chain. McGraw-Hill; 2004.

[4] Redacción APICS. Seis tips para contratar a un operador logístico. Extraído del sitio web www.manufactura.mx a través de apicspuebla.org el 10/08/2018; 2018.

[5] Antún Juan Pablo. Operadores logísticos en México. Extraído del sitio web Énfasis Logística: http://www.logisticamx.enfasis.com/notas/3716-operadores-logisticosmexico el 15/07/2015; 2015

[6] Secretaria de Economía. Directorio De Operadores Logísticos. Extraido del sitio web: http://www.economiasniim.gob.mx/DirLogistico/Consultas.asp el 11/08/2018; 2018.

[7] AOLM Asociación de Operadores Logísticos de México. Extraído del sitio web: http://www.aolm.org.mx/img/op/AOLM\%20\%20\%202015.pdf el 15/7/2015; 2015. 